\title{
A CANONICAL FORM FOR $H$-UNITARY MATRICES
}

\author{
G. J. Groenewald, D. B. Janse van Rensburg and A. C. M. Ran
}

Abstract. In this paper matrices $A$ are considered that have the property that $A^{*} H A=H$, where $H=H^{*}$ is invertible. A canonical form is given for the pair of matrices $(A, H)$ under transformations $(A, H) \rightarrow\left(S^{-1} A S, S^{*} H S\right)$, where $S$ is invertible, in which the canonical form for the $A$-matrix is the usual Jordan canonical form. The real case is studied as well.

Mathematics subject classification (2010): 15A21, 47B50, $15 \mathrm{~B} 57$.

Keywords and phrases: Indefinite inner product space, canonical forms, $H$-unitary matrices.

\section{REFERENCES}

[1] Y.-H. Au-Yeung, C. K. Li, L. Rodman, H-unitary and Lorentz matrices, SIAM J. Matrix Anal. 25, (2004), 1140-1162.

[2] T. YA. AzIzov, I. S. Iohvidov, Linear operators in spaces with an indefinite metric, John Wiley and Sons, Chicester, 1989 [Russian original 1979].

[3] J. BognÁR, Indefinite inner product spaces, Ergebnisse der Mathematik und ihrer Grenzgebiete, Band 78, Springer-Verlag, New York-Heidelberg, 1974.

[4] J. H. Fourie, G. Groenewald, D. B. Janse van Rensburg, A. C. M. Ran, Simple forms and invariant subspaces of $H$-expansive matrices, Linear Algebra and its Applications 470, (2015), $300-340$.

[5] I. Gohberg, P. Lancaster, L. Rodman, Matrices and Indefinite Scalar Products, Oper. Theory: Adv. and Appl. 8, Birkhäuser Verlag, Basel, 1983.

[6] I. Gohberg, P. Lancaster, L. Rodman, Indefinite Linear Algebra and Applications, Birkhäuser Verlag, Basel, 2005.

[7] I. Gohberg, B. Reichstein, Classification of block-Toeplitz H-normal operators, Linear and Multilinear Algebra 34, (1993), 213-245.

[8] B. Huppert, Isometrien von Vektorräumen 1, Arch. Math. 35 (1980), 164-176.

[9] I. S. IOHVIDOV, M. G. KREIN, H. LANGER, Introduction to the spectral theory of operators in spaces with an indefinite metric, Mathematical Research, 9, Akademie-Verlag, Berlin, 1982.

[10] D. B. JANSE VAN REnsBuRg, Structured matrices in indefinite inner product spaces: simple forms, invariant subspaces and rank-one perturbations, Ph. D. thesis, North-West University, Potchefstroom, 2012, http://www.nwu.ac.za/content/mam-personnel.

[11] I. KRUPNIK, P. LANCASTER, $H$-selfadjoint and $H$-unitary matrix pencils, SIAM J. Matrix Anal. Appl. 19, (1998), 307-324.

[12] CHR. MEHL, On classification of normal matrices in indefinite inner product spaces, Electron. J. Linear Algebra 15, (2006), 50-83.

[13] CHR. MEHL, Essential decomposition of normal matrices in real indefinite inner product spaces, Electron. J. Linear Algebra 15, (2006), 84-106.

[14] V. Mehrman, H. XU, Structured Jordan canonical forms for structured matrices that are Hermitian, skew Hermitian or unitary with respect to indefinite inner products, Electron. J. Linear Algebra 5, (1999), 67-103.

[15] J. Milnor, On Isometries of Inner Product Spaces, Inventiones Math. 8, (1969), 83-97.

[16] L. Rodman, Similarity vs unitary similarity: Complex and real indefinite inner products, Linear Algebra and its Applications 416, (2006), 945-1009.

[17] V. V. SERGEICHUK, Classification problems for systems of forms and linear mappings, Math. USSRIzv. 31, (1988), 481-501 (translation from Russian). 
[18] V. V. SERGEICHUK, Canonical matrices of isometric operators on indefinite inner product spaces, Linear Algebra and its Applications 428, (2008), 154-192.

[19] L. S Mith, Linear Algebra, Springer-Verlag, New York, 1984. 